\title{
'We have all had to adapt'
}

\section{align}

\author{
The ADAPT service is an expert and independent fee-based business consulting service offered by Align Technology \\ to optimise practices' operational workflow and processes to enhance patients' experiences and customer and staff \\ satisfaction, which can translate into higher growth and greater efficiencies for orthodontic practices.
}

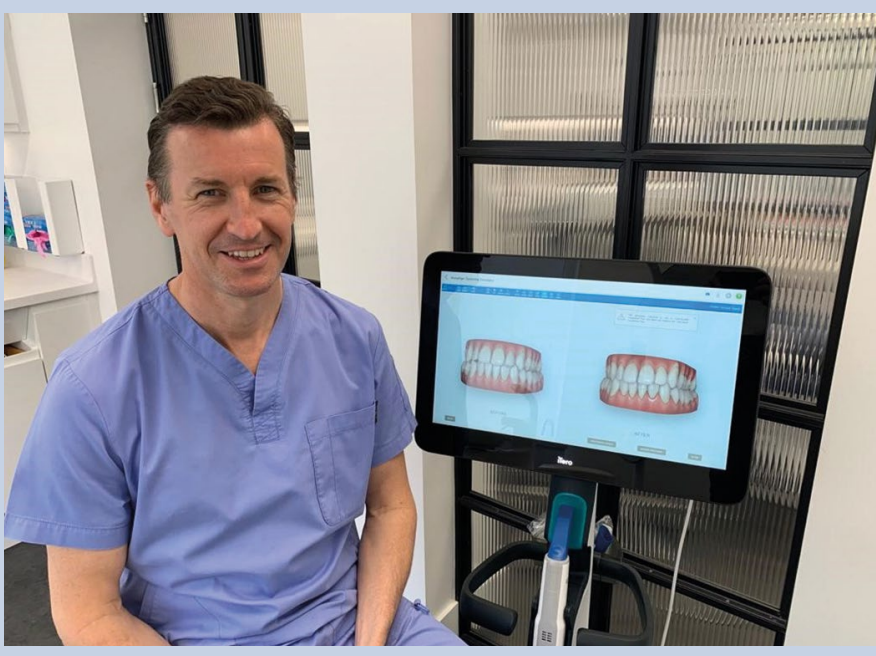

Dr Rhu McKelvey

\section{Exciting and challenging}

According to Dr Rhu McKelvey: 'Since I started Beam Orthodontics in Dundee 13 years ago, I have invested in dental business coaches. Align Technology's ADAPT service is different and is offered to orthodontists looking for practice optimisation and digitisation, and provides a very focused, different and evidence-driven approach to practice development. It appealed to me that we would be taking a deep-dive into the practice numbers and seeing what was really going on with orthodontic treatments, and more specifically, focusing on clear aligner therapy. I liked the idea of having a third party analysing what we were doing and giving me constructive feedback. I felt it was both exciting and challenging, and I wanted some truths that I didn't know, or had ignored.

'The ADAPT service helped me embrace and adopt a more digital workflow. I was on that path already but it certainly catalysed and accelerated this aspect of the practice and helped focus my attention, as well as my team's on fast-tracking a technological approach. With the insights I have gleaned from the ADAPT service, I have been able to move forward more consistently and faster over the last 18 months. In a time when it would have been considered wise to mothball plans for growth we have expanded considerably; we doubled the physical size of the practice by occupying the ground floor of our building in addition to the existing practice upstairs and added four more surgeries going from five to nine.'

\section{Growth on all fronts}

'We have also taken on a new specialist orthodontist, an additional manager, more nurses and receptionists, and we had just started training an additional orthodontic therapist. So growth on all fronts. We have also introduced more iTero intraoral scanners. As a result, our Invisalign numbers have grown steadily since we re-opened and the new separate space has allowed me to focus on this part of the business, helping me to become a Diamond Invisalign provider.

'We have had to adapt and change the way we work as a result. We modified a lot of our processes and became much more treatment coordinator focused. Historically I had been the bottleneck in new patient assessment and starts, so we have refined things to address that and get me out of the way. You go on a course and hear some things and agree they are great ideas, but to follow them through and make them happen and make things stick is a different challenge. The ADAPT approach helped the follow through, getting the rest of the team on board a new approach, and it has made a massive difference. ADAPT provided me with the follow through and the execution strategy which seems to be lacking for many orthodontic practices.'

\section{Taking a digital approach}

Dr McKelvey says: 'I have used the iTero intraoral scanner for over three years. My third new one arrived a few months ago. The value of the scanner when treating Invisalign patients has been immense; it is a total game changer and I would not dream of going back to not having a scanner for conducting Invisalign treatments. Align Technology has worked very closely with Systems for Dentists (SfD) to capture photos and digital study models and upload them directly onto the SfD practice software. This integration has saved us time and money with our record collection and management of new patients.

'The beauty of the iTero intraoral scanner integration with $\mathrm{SfD}$ is that when you take a scan, the data is saved and automatically sent to SfD without any input from staff. It saves all hassle of uploading and downloading, you can't lose anything, you can't make mistakes, it pulls the two together and is a huge time saver. It is the twentyfirst century solution.'

\section{The future of orthodontics}

Dr McKelvey adds: 'I did my original Invisalign certification course around 2005; however, the Invisalign system is a completely different technology from what it was when I first certified. Anyone who has previously trained but not developed their skills, I would strongly encourage them to open that door again because it is the future of orthodontics, it is here to stay, has great advantages in many situations, and is a good treatment for most of the cases I treat. If you told me that five years ago, I would not have believed you. I used to do $90 \%$ fixed braces and now I do $80 \%$ Invisalign cases and $20 \%$ fixed'.

To find out about upcoming Invisalign certification courses for orthodontists please visit https://www.yourbrillianceenhanced. com/events. 